\title{
Bubble Nucleation and Growth of Dissolved Gas in Solution Flowing Across a Cavitating Nozzle
}

\author{
BAN Zhen Hong ${ }^{1, a}$, LAU Kok Keong ${ }^{2, b^{*}}$, AZMI Mohd Shariff ${ }^{3, c}$ \\ ${ }^{1,2,3}$ Chemical Engineering Department, Universiti Teknologi PETRONAS, \\ Bandar Seri Iskandar, 31750 Tronoh, Perak, Malaysia. \\ azhbanzh@gmail.com, ${ }^{b}$ laukokkeong@petronas.com.my, ${ }^{c}$ azmish@petronas.com.my
}

Keywords: Supersaturated, Bubble Formation, Nozzle

\begin{abstract}
Computational modelling of dissolved gas bubble formation and growth in supersaturated solution is essential for various engineering applications, including flash vaporisation of petroleum crude oil. The common mathematical modelling of bubbly flow only caters for single liquid and its vapour, which is known as cavitation. This work aims to simulate the bubble nucleation and growth of dissolved $\mathrm{CO}_{2}$ in water across a cavitating nozzle. The dynamics of bubble nucleation and growth phenomenon will be predicted based on the hydrodynamics in the computational domain. The complex interrelated bubble dynamics, mass transfer and hydrodynamics was coupled by using Computational Fluid Dynamics (CFD) and bubble nucleation and growth model. Generally, the bubbles nucleate at the throat of the nozzle and grow along with the flow. Therefore, only the region after the throat of the nozzle has bubbles. The bubble nucleation rate is significant in between normalised position of 3 and 4 , with highest rate at about $1.2 \times 10^{26}$ nuclei $/ \mathrm{m}^{3} \cdot \mathrm{s}$. Similarly, the bubble growth rate is significant in normalised position of 2 and 4, with highest rate recorded at about $0.09 \mathrm{~m} / \mathrm{s}$. This approach is expected to be useful for various types of bubbly flow modelling in supersaturated condition.
\end{abstract}

\section{Introduction}

Bubbles can be found in daily life easily, either it is created naturally or used in various engineering application. The bubbles are formed when the equilibrium system is disturbed. Generally, there are three types of bubble formation; vapour bubbles caused by boiling or cavitation and dissolved gas bubbles caused by depressurisation. Bubbles will undergo nucleation, growth, coalescence and breakage in bubbly flow system.

There are several works had been carried out to study the bubble nucleation process. The Classical Nucleation Theory (CNT) has stated that a critical cluster of bubble can only be formed when the energy obtained by the molecules had exceeded the energy barrier [1]. However, most of the numerical studies in bubbly flow system had employed a presumed bubble nuclei density in the computational domain $[2,3]$. In other words, their simulation has ignored the bubble nucleation process and started the bubble growth simulation with pre-existed bubble nuclei. There were several numerical studies on cavitation flow had attempted to incorporate the nucleation model into the simulation. Delale et al. [4] had included the bubble nucleation model in the simulation of cavitating flow across a nozzle. From the work carried out by Delale et al. [4], the bubble nucleation was found only occurred near to the throat of the nozzle. This was due to sufficient pressure drop at the throat of the nozzle.

The bubble nuclei will grow after its formation to bring the system back to equilibrium state. Several experimental and theoretical studies on bubble growth in supersaturated solution had been carried out $[5,6]$. Basically, the modelling of bubble growth model had been divided into two types; origin from slightly or strongly supersaturated solution. The Scriven model is commonly used to describe the bubble growth in slightly supersaturated solution such as $\mathrm{CO} 2$ bubble grows in carbonated drink [7]. On the other hand, the model is often employed to model bubble growth in strongly supersaturated solution including bubble grows in magma [8]. Since the bubble growth in 
supersaturated solution is difficult due to the needs to couple hydrodynamics, mass transfer and, the numerical studies on bubble growth was limited. There are also several computational simulation works on bubble growth in supersaturated solution by using Lattice Boltzmann method [9]. However, this method has suffered several limitations such as meshing and numerical instability.

The numerical estimation of bubbly flow in supersaturated solution is important as it can be applied to various engineering application. However, the simulation works including nucleation and bubble growth model for the bubbly flow in supersaturated solution is scarcely available. The bubble growth dynamics is difficult to be accomplished by using classical Computational Fluid Dynamics (CFD) approach. Therefore, the main objective of current work is to simulate the $\mathrm{CO}_{2}$ bubble nucleation and growth phenomenon across a nozzle. This was accomplished by coupling the bubble nucleation and growth model together with the hydrodynamics equations in CFD and population balance model (PBM).

\section{Numerical Method}

The commercial software ANSYS FLUENT 15.0 was employed to simulate the bubble nucleation and growth phenomenon for water supersaturated with dissolved $\mathrm{CO}_{2}$ flowing across a cavitating nozzle. The normalized area of the nozzle, $\mathrm{A}(\mathrm{x})$, was given by

$$
\begin{aligned}
& A(x)=\left\{1+0.5\left[1-\cos \left(\frac{2 \pi x}{5}\right)\right]\right\}^{-1 / 2} \\
& \text { for } 0 \leq \mathrm{x} \leq 5
\end{aligned}
$$

with $\mathrm{x}$ as the length of the nozzle. The throat of the nozzle shall be located at $\mathrm{x}=2.5$, which is the middle of the nozzle, as shown in Fig. 1.

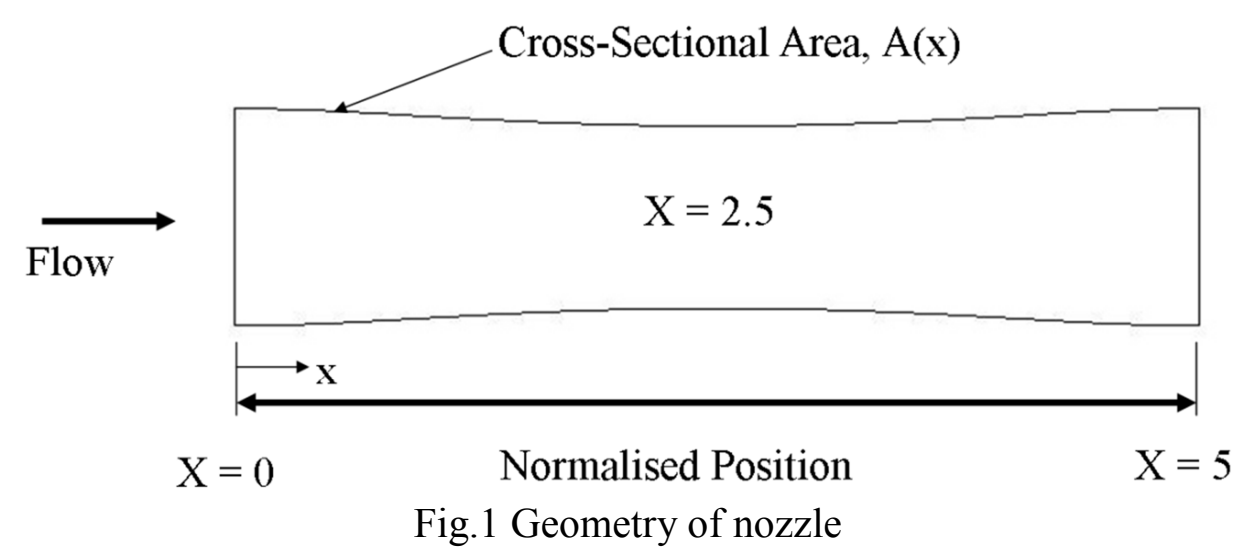

Fig. 2 shows the mesh of the computational domain in current work. The mesh is generated based on quadrilaterals method. A total of 16000 cells were generated in the computational domain based on the grid independency test, by comparing with computational domain with 1200 and 28500 cells. The result obtained from 16000 cells domain was found to be similar with the 28500 cells domain. Therefore, 16000 cells domain was chosen in current work. Volume finite method was employed for the discretization of equations in the simulation. The solution was solved by using coupled algorithm. The well-known k- $\varepsilon$ turbulence model was used to cater for the turbulence effect in the simulation. 


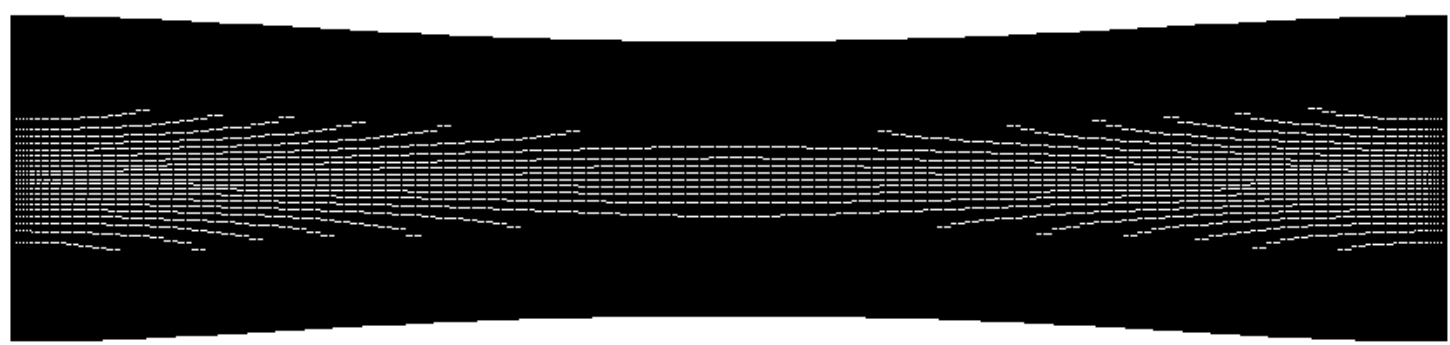

Fig. 2: Mesh of the computational domain

The simulation case for water saturated with $\mathrm{CO}_{2}$ at initial pressure of 2 bar flowing across the nozzle and exit at atmospheric pressure. Therefore, the pressure inlet was set at 2 bar at temperature of $293.15 \mathrm{~K}$. The $\mathrm{k}-\varepsilon$ turbulence model was employed in the CFD solver to simulate the fluid flow in the computational domain.

In current work, the Classical Nucleation Theory (CNT) was used as the bubble nucleation mathematical model to describe the bubble nucleation process. The bubble growth dynamics as suggested by Gor et al. [10] was used to describe the bubble growth phenomenon. These models were incorporated with the population balance model (PBM) to estimate the bubble nucleation and growth phenomenon in the domain. In addition, the Quadrature Method of Moments (QMOM) was implemented as the solution method for the PBM.

The governing equations for hydrodynamics employed in current work are the continuity and momentum equations as shown in Eqn. (2) and (3).

Mass conservation

$\frac{\partial \rho}{\partial t}+\nabla \cdot(\rho \boldsymbol{u})=0$

Momentum conservation

$\rho\left(\frac{D \mathbf{u}}{D t}\right)=-\nabla p+\mu \nabla^{2} \mathbf{u}+\mathbf{f}$

The number density of bubble nuclei formed and flow in the domain was handled by PBM as

$$
\frac{\partial n(\mathrm{~V}, t)}{\partial t}+\nabla \cdot[\boldsymbol{u} n(V, t)]=-\frac{\partial}{\partial V}[J(V) n(V, t)]
$$

\section{Results and Discussion}

The bubble nucleation and growth of dissolved $\mathrm{CO}_{2}$ in water across a nozzle has been simulated. The hydrodynamics in the nozzle is the key to determine the bubble nucleation and growth rate. The hydrodynamics in the nozzle was affected by the bubble growth and changed accordingly. Therefore, the couplings of hydrodynamics and bubble nucleation and growth equations are essential to model the bubble growth phenomenon in the nozzle.

Bubble Nucleation Rate. The bubble nucleation rate computed along the axis of the nozzle is presented in Fig. 3. From the figure, the significant bubble nucleation rate is found to be near to the throat of the nozzle. However, the graph is slightly skewed towards the outlet due to the formation of $\mathrm{CO}_{2}$ bubbles. Since the pressure drop at the throat of the nozzle is sufficient to initiate the bubble nucleation, the critical cluster of bubble nuclei would gain enough energy to be formed. Therefore, the bubble nucleation rate estimated has been found to be highest at the wall of the throat of the nozzle. After the bubble nuclei have formed, they would flow according to the hydrodynamics in the nozzle. 


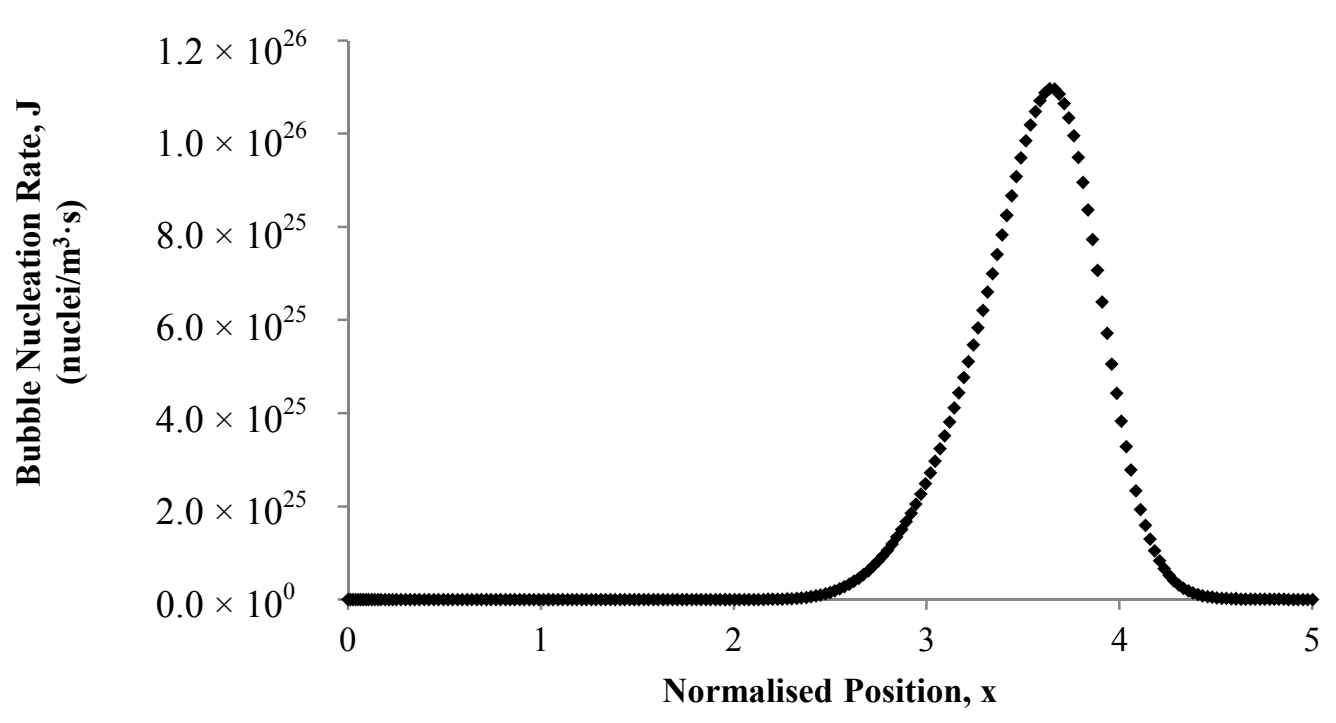

Fig. 3 Bubble nucleation rate along the axis of the nozzle

Bubble Growth. After the bubble nuclei have formed, they will grow according to the supersaturation ratio which they have experienced in the nozzle. The solution will become supersaturated with dissolved $\mathrm{CO}_{2}$ when the pressure has been lowered. Thus, the solution was in a non-equilibrium state. In order to restore the equilibrium system, the dissolved $\mathrm{CO}_{2}$ in the solution have to be reduced. Hence, the dissolved $\mathrm{CO}_{2}$ will transform into bubbles and reduce the amount of dissolved $\mathrm{CO}_{2}$ in the solution. Meanwhile, the bubble will grow as the dissolved $\mathrm{CO}_{2}$ has transferred into the gas phase (bubbles). Fig. 4 shows the bubble growth rate in the nozzle. Generally, larger pressure drop will generate larger supersaturation ratio. The mass transfer rate or the bubble growth rate was determined by the supersaturation ratio. Therefore, the bubble growth rate was higher at the larger pressure drop region.

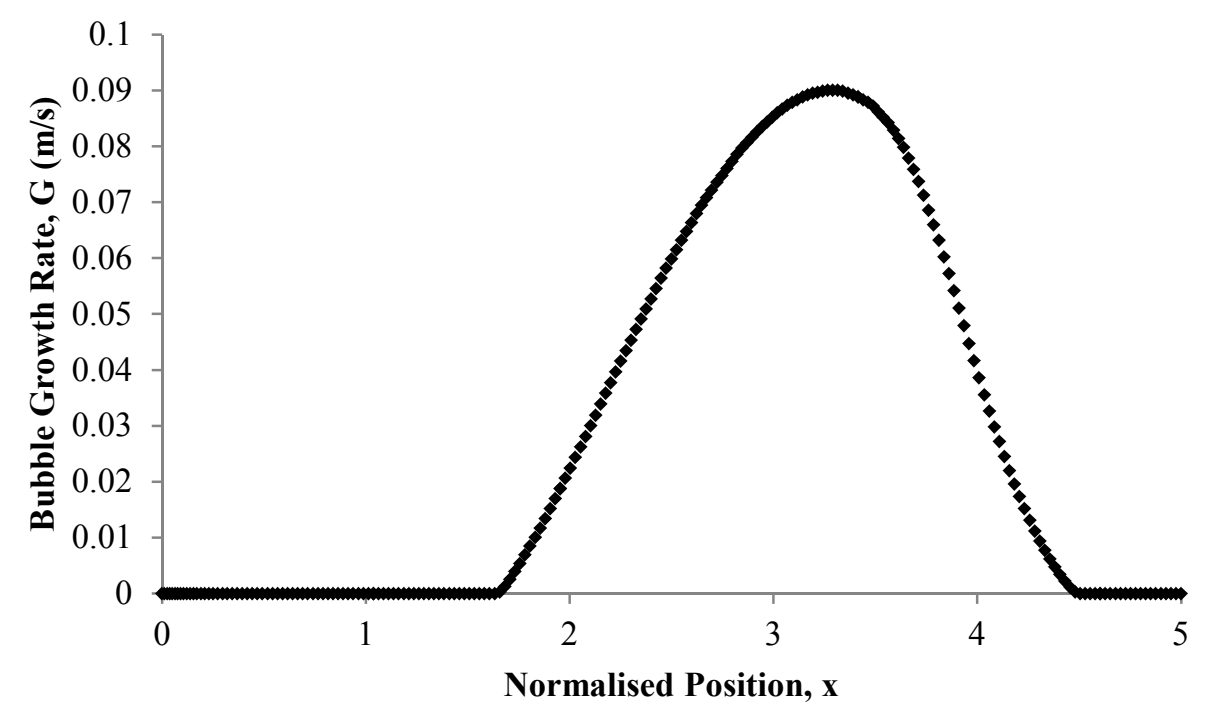

Fig. 4 Bubble growth rate along the axis of the nozzle

Bubble Volume Fraction. Since the density of $\mathrm{CO}_{2}$ bubbles and water solution is different significantly, the small amount of bubbles will occupy a relatively large space in the nozzle. The volume fraction of the $\mathrm{CO}_{2}$ bubbles is presented in Fig. 5. From the figure, the $\mathrm{CO}_{2}$ bubbles have occupied a relatively significant volume in the region after the throat of the nozzle. Higher volume 
fraction of $\mathrm{CO}_{2}$ indicates that the $\mathrm{CO}_{2}$ bubbles formed has occupied more space at the specific region.

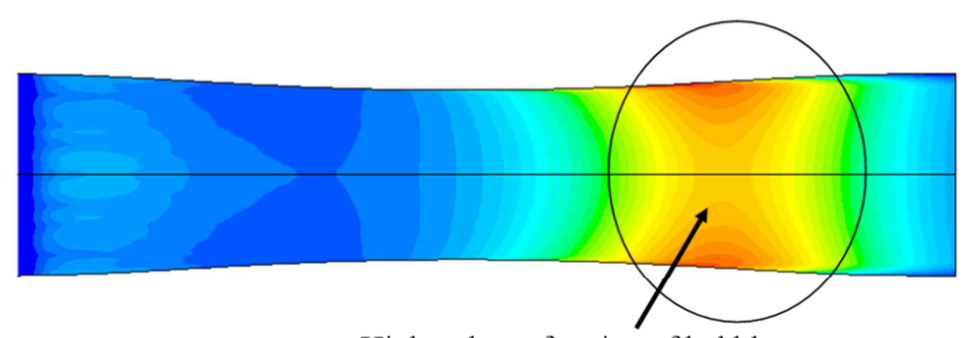

High volume fraction of bubbles

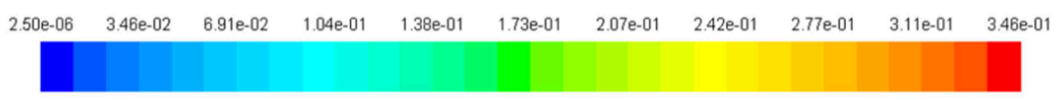

Fig. 5 Volume fraction of $\mathrm{CO}_{2}$ bubbles in the nozzle

\section{Conclusion}

The bubble growth phenomenon of a supersaturated $\mathrm{CO}_{2}$-water solution across a nozzle has been successfully simulated. The bubble nucleation and growth model have been coupled with the hydrodynamics and mass transfer equations by using Population Balance Model (PBM). The bubble nucleation rate and growth rate was found to be highest at the throat of the nozzle. The bubble nuclei has grown across the nozzle and occupied a relatively significant volume in the nozzle after the throat. This method has enabled the prediction of bubble nucleation rate and growth rate of supersaturated solution in the computational domain.

\section{References}

[1] Lubetkin, S.D., The fundamentals of bubble evolution, Chemical Society Reviews. 24(1995) 243-250.

[2] Preston, A.T., T. Colonius, and C.E. Brennen, A numerical investigation of unsteady bubbly cavitating nozzle flows, Physics of Fluids. 14(2002) 300-311.

[3] Moholkar, V.S. and A.B. Pandit, Numerical investigations in the behaviour of one-dimensional bubbly flow in hydrodynamic cavitation, Chemical Engineering Science. 56(2001) 1411-1418.

[4] Delale, C.F., K. Okita, and Y. Matsumoto, Steady-State Cavitating Nozzle Flows With Nucleation, Journal of Fluids Engineering. 127(2005) 770-777.

[5] Kuchma, A.E., G.Y. Gor, and F.M. Kuni, Stages of steady diffusion growth of a gas bubble in strongly supersaturated gas-liquid solution, Colloid Journal. 71(2009) 520-528.

[6] Frank, X., N. Dietrich, J. Wu, R. Barraud, and H.Z. Li, Bubble nucleation and growth in fluids, Chemical Engineering Science. 62(2007) 7090-7097.

[7] Barker, G.S., B. Jefferson, and S.J. Judd, The control of bubble size in carbonated beverages, Chemical Engineering Science. 57(2002) 565-573.

[8] Gor, G.Y. and A.E. Kuchma, Dynamics of gas bubble growth in a supersaturated solution with Sievert's solubility law, The Journal of Chemical Physics. 131(2009) 034507-7.

[9] Chen, X.-P., C.-W. Zhong, and X.-L. Yuan, Lattice Boltzmann simulation of cavitating bubble growth with large density ratio, Computers \& Mathematics with Applications. 61(2011) 35773584.

[10]Gor, G.Y., A.E. Kuchma, and F.M. Kuni, Gas Bubble Growth Dynamics in a Supersaturated Solution: Henry's and Sievert's Solubility Laws, Nucleation Theory and Applications, (2012) 213-233. 